\title{
Methamphetamine-induced structural plasticity in the dorsal striatum
}

\author{
Jakub P. Jedynak, ${ }^{1}$ Jason M. Uslaner, ${ }^{2}$ José A. Esteban ${ }^{1,3}$ and Terry E. Robinson ${ }^{1,2}$ \\ ${ }^{1}$ Neuroscience Program, The University of Michigan, Ann Arbor, MI, USA \\ ${ }^{2}$ Department of Psychology (Biopsychology), The University of Michigan, Ann Arbor, MI, USA \\ ${ }^{3}$ Department of Pharmacology, The University of Michigan, Ann Arbor, MI, USA
}

Keywords: addiction, amphetamine, basal ganglia, dendrites, morphology, spines

\begin{abstract}
Repeated exposure to psychostimulant drugs produces long-lasting changes in dendritic structure, presumably reflecting a reorganization in patterns of synaptic connectivity, in brain regions that mediate the psychomotor activating and incentive motivational effects of these drugs, including the nucleus accumbens and prefrontal cortex. However, repeated exposure to psychostimulant drugs also facilitates a transition in the control of some behaviors from action-outcome associations to behavior controlled by stimulus-response (S-R) habits. This latter effect is thought to be due to increasing engagement and control over behavior by the dorsolateral (but not dorsomedial) striatum. We hypothesized therefore that repeated exposure to methamphetamine would differentially alter the density of dendritic spines on medium spiny neurons (MSNs) in the dorsolateral vs. dorsomedial striatum. Rats were treated with repeated injections of methamphetamine, and 3 months later dendrites were visualized using Sindbis virusmediated green fluorescent protein (GFP) expression in vivo. We report that prior exposure to methamphetamine produced a significant increase in mushroom and thin spines on MSNs in the dorsolateral striatum, but a significant decrease in mushroom spines in the dorsomedial striatum. This may be due to changes in the glutamatergic innervation of these two subregions of the dorsal striatum. Thus, we speculate that exposure to psychostimulant drugs may facilitate the development of S-R habits because this reorganizes patterns of synaptic connectivity in the dorsal striatum in a way that increases control over behavior by the dorsolateral striatum.
\end{abstract}

\section{Introduction}

Long-lasting changes in behavior and psychological function that accrue with experience are thought to be due to a reorganization of synaptic connections, altering the operation of affected cells and circuits (Ramón y Cajal, 1894; Hebb, 1949). This is manifest in part by structural plasticity, including changes in patterns of dendritic branching and in the density of dendritic spines (Greenough et al., 1990; Kolb \& Whishaw, 1998; Rosenzweig, 2003). For example, experience with a number of different drugs of abuse induces structural plasticity, which may contribute to some of the long-term behavioral and psychological consequences of drug use, including addiction (Robinson \& Kolb, 2004).

To date, most research on drug experience-dependent structural plasticity has focused on brain regions that mediate the psychomotor activating and incentive motivational effects of drugs, especially the ventral striatum (nucleus accumbens) and medial prefrontal cortex. Repeated exposure to D-amphetamine or cocaine, whether experimenter-administered or self-administered, produces a persistent increase in dendritic branching and spine density on medium spiny neurons (MSNs) in the shell and core of the nucleus accumbens and on pyramidal cells in the medial prefrontal cortex (Robinson \& Kolb, 2004). However, another brain region that changes as a function of experience with psychostimulant drugs is the dorsal striatum

Correspondence: Dr T.E. Robinson, ${ }^{2}$ Department of Psychology (Biopsychology), as above.

E-mail: ter@umich.edu

Received 21 July 2006, revised 21 November 2006, accepted 22 November 2006
(Paulson \& Robinson, 1995; Canales \& Graybiel, 2000; Porrino et al., 2004). Indeed, this may be related to one well-documented symptom of addiction, the development of 'automatized' stimulus-response (S-R) habits, which is thought to reflect a transfer of control over behavior from the ventral to the dorsal striatum (Tiffany, 1990; Berke \& Hyman, 2000; Everitt et al., 2001).

Little is known about the extent to which drugs of abuse alter dendritic morphology in the dorsal striatum. The primary purpose of this study therefore was to characterize the effect of repeated exposure to methamphetamine on the density of dendritic spines on MSNs in the dorsal striatum. However, the dorsal striatum is anatomically and functionally heterogeneous. For example, the dorsolateral (but not dorsomedial) striatum is important for habit (S-R) learning (Yin et al., 2004, 2006). Although the role of S-R habits in the etiology of addiction is debated (Robinson \& Berridge, 2003), it is clear that repeated exposure to amphetamine or cocaine can facilitate the transition from behavior controlled by action-outcome associations to behavior controlled by S-R habits (Miles et al., 2003; Nelson \& Killcross, 2006), and that S-R habits characterize 'automatized' patterns of drug consumption behavior in addicts (Tiffany, 1990). We hypothesized therefore that repeated exposure to amphetamine would have different effects on synaptic connectivity (assessed by spine density) in the dorsolateral vs. the dorsomedial striatum. In addition, the primary form of amphetamine currently used illicitly is methamphetamine, but in all previous studies on amphetamine-induced structural plasticity D-amphetamine was given. Therefore, the methylated form of amphetamine was used here. Finally, a new approach was used to visualize spines, Sindbis virus-mediated GFP expression 
(Maletic-Savatic et al., 1999; Lendvai et al., 2000), which also allowed us to determine whether previous findings were unique to Golgi-Cox staining.

\section{Materials and methods Subjects}

Adult female Sprague-Dawley rats weighing 220-280 g were housed three per cage $(45.4 \times 23.8 \times 20.3 \mathrm{~cm})$ on a $14: 10 \mathrm{~h}$ light : dark cycle with food and water available ad libitum. Female rats were used because amphetamine-induced behavioral and structural plasticity in the ventral striatum has been shown to persist for at least 3.5 months in female rats (Robinson, 1984; Paulson et al., 1991; Kolb et al., 2003; also see Hamamura et al., 1991). All experimental procedures were approved by the University of Michigan Committee on the Use and Care of Animals following National Institute of Health guidelines.

\section{Methamphetamine pretreatment}

On treatment days rats were transported from their home cages and placed individually into locomotor activity monitors $(41 \times 25.4 \times 20.3 \mathrm{~cm})$ that had a rectangular clear Plexiglas insert $(23 \times 6.3 \times 20.3 \mathrm{~cm})$ forming a pathway around the perimeter of the cage where the animals could walk. Infrared photocells surrounding the perimeter of each chamber recorded beam breaks, which were used as an index of locomotor activity. Following $30 \mathrm{~min}$ of habituation, rats received an injection of either saline $(N=4)$ or methamphetamine $\mathrm{HCl}(N=7)$ for five consecutive days followed by two nondrug days for four consecutive weeks. The initial injection for each week was $0.5 \mathrm{mg} / \mathrm{kg}$ of methamphetamine $\mathrm{HCl}$, and the remaining weekly injections were given using an escalating-dose regimen adapted from Paulson et al. (1991). Specifically, the doses were gradually increased until animals received $6 \mathrm{mg} / \mathrm{kg}$ methamphetamine $\mathrm{HCl}$ (weight of the salt) over the last four days of the fourth week. An additional (and last) injection of $0.5 \mathrm{mg} / \mathrm{kg}$ was given 3 days later, in the activity monitors, and then the animals were left undisturbed in their home cages for 3 months. This procedure resulted in a significant increase in locomotor activity between the first and the last test session ( $t=3.864 ; P=0.008$; data not shown).

\section{Virus production}

pEGFP-C1 was digested with Apa I and Nhe I, and cloned into wildtype pSinRep5 vectors. Ligation products were transformed into competent cells and positive colonies were identified through restriction enzyme digest. Viral constructs and a separate defective helper construct, which encodes for structural proteins that are required for viral particle packaging, were linearized with Not I. RNA was generated and electroporated into baby hamster kidney cells. Viral particles were harvested $36 \mathrm{~h}$ later.

\section{Viral infection}

Three months after the last injection of saline or methamphetamine, the animals were anesthetized using a cocktail of ketamine hydrochloride (Fort Dodge Animal Health, Fort Doge, IA, USA) and xylazine hydrochloride (Lloyd Laboratories, Shenandoah, IA, USA), and placed into a stereotaxic instrument (David Kopf Instruments, Tujunga, CA, USA). Infusion cannulae (27-gage) were lowered bilaterally into the head of the caudate-putamen (anterior from bregma
$1.2 \mathrm{~mm}$; lateral from the midline $2.6 \mathrm{~mm}$; ventral from the skull surface $5.0 \mathrm{~mm}$ ) over 30-60 s until the desired depth was reached, and then they were retracted $0.1 \mathrm{~mm}$ before starting the infusion. GFP viral particles were injected at $0.2 \mu \mathrm{L} / \mathrm{min}$ over $10 \mathrm{~min}$, and allowed to diffuse for $3 \mathrm{~min}$ before the infusion cannulae were raised. Twentyfour hours following surgery, animals were given an overdose of Fatal-Plus ${ }^{\circledR}$ Solution (pentobarbital; Vortech Pharmaceuticals, Dearborn, MI, USA) and perfused transcardially with $4 \%$ paraformaldehyde in $1 \times$ phosphate-buffered saline (PBS; pH 7.4) at $30 \mathrm{~mL} / \mathrm{min}$ for $5 \mathrm{~min}$. Brains were postfixed in perfusion medium for $2 \mathrm{~h}$ and then placed in $30 \%$ sucrose in $1 \times$ PBS solution overnight. Following sucrose infiltration, $120-\mu \mathrm{m}$ sections were prepared on a cryostat and stored in PBS. Sections were immediately mounted on slides, coverslipped with Prolong Gold Antifade Reagent (Invitrogen, Carlsbad, CA, USA) and stored at $4{ }^{\circ} \mathrm{C}$.

\section{Confocal microscopy}

GFP-expressing MSNs in the left and right dorsal striatum were visualized using an Olympus FV-500 confocal microscope. Initially, GFP-expressing cells were identified using $20 \times$ air objective lens (NA 0.7). Cells that were not immediately adjacent to the injection site, showed well-defined GFP labeling, and appeared to have a number of full-length dendrites within the plane of the section were then selected. Neurons near the site of injection were not analysed due to concern over the potential influence of needle tract damage on neuronal morphology. Next, the tips of dendritic branches on a given cell, that appeared to be terminal tips, were visualized using a $60 \times$ oil immersion objective lens (NA 1.4). If the dendrite could be traced all the way back to the soma, and if it indeed had a thirdorder or above terminal tip, confocal imaging was initiated from the terminal tip of the dendrite and continued in a retrograde fashion towards the cell body. Confocal stacks consisted of 31-191 sections at $0.23 \mu \mathrm{m}$ in thickness imaged with a $z$-step of $0.1 \mu \mathrm{m}$. At a zoom factor of 2.5 and XY resolution of $0.17 \mu \mathrm{m}$, images were generated with 12 pixels $/ \mu \mathrm{m}$ (a resolution more than adequate for imaging spines). This procedure was then repeated on the same cell until it was too photo-bleached to obtain further images. Typically, images of one-four dendrites were obtained per cell. Another cell was then identified and the process repeated, and images were obtained from one-five cells/hemisphere.

For cell body reconstructions whole neurons were imaged with a $20 \times$ air objective lens (NA 0.7). Confocal stacks consisted of 56-109 images with a $z$-axis resolution of $0.425 \mu \mathrm{m}$. At zoom factors between 1 and 2 and an XY resolution of $0.38 \mu \mathrm{m}$, images with 1.6-3.2 pixels $/ \mu \mathrm{m}$ were generated. Some cell bodies were imaged with sections greater than $1 \mu \mathrm{m}$ in thickness, although no difference existed in the XY resolution leaving analysis in the $x y$ plane unaffected.

\section{Analysis}

All quantitative analysis was conducted using Neurolucida ${ }^{\circledR}$ (Microbrightfield, Williston, Vermont, USA) software equipped with a confocal module on a custom-built computer. First, the confocal stacks obtained in a given cell were examined, and it was confirmed that the stack contained a third-order or above terminal branch that was at least $50 \mu \mathrm{m}$ in length. Those that did not were discarded. Next, the stack with the most intense GFP signal throughout the length of the dendrite, and with spines best resolved, was selected for detailed analysis. First, the entire dendrite was traced and its length was calculated using the neuron-tracing feature in Neurolucida ${ }^{\circledR}$. Next, 
beginning at the tip of the dendrite and moving towards the soma, spines were counted by using Neurolucida ${ }^{\circledR}$ to place a marker for each spine along the length of the dendrite, and each branch point was also identified using a different marker. Only protuberances with a clear connection from the head of the spine to the shaft of the dendrite were counted as spines. The confocal stack being analysed with Neurolucida $^{\circledR}$ was viewed simultaneously on a second monitor using Olympus Fluoview ${ }^{\circledR}$ software. Briefly, images in Fluoview ${ }^{\circledR}$ were converted to gray-scale and intensity adjustments were made to enhance the images. When spines were in close proximity this second enhanced image was used to make judgments about the number and type of spines. An example of such an enhanced image is shown in Fig. 3. Neuroexplorer $^{\circledR}$ was then used to calculate spine density, which is expressed as spines per $10 \mu \mathrm{m}$. Only one terminal branch per cell was analysed. We selected spines on third-order and higher terminal dendrites for analysis because in our previous studies we consistently found the largest effects of drugs on this particular measure, and it occurred on distal (but not proximal) dendrites (Li et al., 2003; Robinson \& Kolb, 2004).

For the analysis of different types of spines, individual spines were divided into four distinct classes based on spine head diameter $\left(d_{\mathrm{h}}\right)$ and the presence or absence of a discernible spine neck (Harris et al., 1992). A circular template with a diameter of $0.55 \mu \mathrm{m}$ was placed over the head of the spine, and the extent to which any part of the spine head extended over the boundaries of the template allowed the observer to distinguish between 'thin' and 'mushroom' or 'stubby' and 'chubby' spines, using the following criteria. Spines with no discernible neck were defined as either 'chubby' or 'stubby' based on $d_{\mathrm{h}}$. In 'stubby' spines $d_{\mathrm{h}}<0.55 \mu \mathrm{m}$, and in 'chubby' spines $d_{\mathrm{h}}>0.55 \mu \mathrm{m}$. Spines with a discernible neck were defined as either 'thin' or 'mushroom', based on $d_{\mathrm{h}}$. In 'thin' spines $d_{\mathrm{h}}<0.55 \mu \mathrm{m}$ and in 'mushroom' spines $d_{\mathrm{h}}>0.55 \mu \mathrm{m}$.

For cell body analysis, a perimeter was traced along the outer edge of the cell body from each image in the confocal stack. The largest perimeter $(\mu \mathrm{m})$ from each cell body was used as an indicator of cell body size.

Data were obtained from a total of 42 cells in 11 individual rats, and each neuron was considered an independent measure for statistical analyses. Group differences were determined by a two-way ANOVA (treatment by brain region) followed by Bonferroni-corrected post hoc tests. Note that the experimenter who conducted the analysis (J.P.J.) was blind to treatment condition during all phases of image acquisition and analysis.

\section{Results}

\section{Effect of methamphetamine on spine density in the dorsolateral} vs. dorsomedial striatum

Figure 1A illustrates the typical pattern of infection seen in the dorsal striatum $24 \mathrm{~h}$ following injection of the Sindbis virus. Many labeled cells are evident away from the injection site (Fig. 1B), and a representative cell is shown in Fig. 1C. Figure 1D shows the location of all the neurons in the dorsolateral and dorsomedial striatum that were analysed. Figure 2 illustrates, at higher magnification, the quality of the images used to quantify dendritic spines. Figure 3 shows the effect of exposure to methamphetamine 3 months earlier on the total number of dendritic spines on third-order or above terminal branches of MSNs in the dorsolateral and the dorsomedial striatum. There was a significant effect of methamphetamine treatment that varied by brain region, as indicated by a significant treatment by brain region interaction $(F=17.75, P<0.0001)$. Post hoc tests showed that
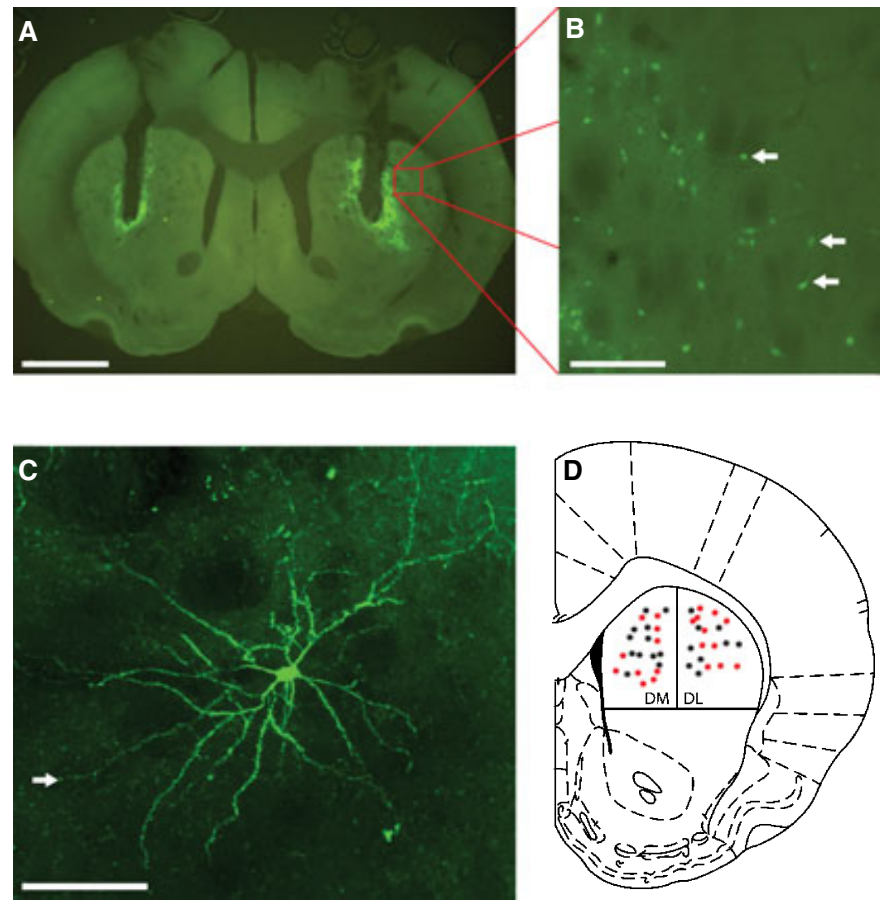

FIG. 1. An illustration of GFP-expressing neurons following Sindbis virus infection. (A) Coronal slice displaying the typical infection pattern $24 \mathrm{~h}$ following viral infusion. Scale bar: $2 \mathrm{~mm}$. (B) Magnified view of the boxed region in (A). Note that the intensity of infection decreases away from the site of infection. Possible candidates for analysis are marked by white arrows. Scale bar: $200 \mu \mathrm{m}$. (C) Confocal stack of a MSN in the caudate putamen. The white arrow marks a third-order or higher terminal dendrite that was selected for analysis. Scale bar: $100 \mu \mathrm{m}$. (D) The boundaries for the dorsomedial (DM) and dorsolateral (DL) areas of the caudate putamen that were analysed, and the location of individual cells selected for analysis. Black circles indicate the location of cells in the saline group and red circles cells in the methamphetamine group. Cells were collapsed from both hemispheres and sections $+0.7-1.7 \mathrm{~mm}$ anterior to bregma.

methamphetamine treatment increased spine density in the dorsolateral striatum by an average of $17.9 \%(P=0.0005$; Fig. 3A), but decreased spine density in the dorsomedial striatum by an average of $11.6 \%$ $(P=0.031$; Fig. 4B). There was also a regional difference in spine density, in that in the spine density of saline-treated animals was significantly higher in the dorsomedial than in the dorsolateral striatum $(P=0.001)$.

There was no effect of methamphetamine on the size of the cell bodies of the MSNs sampled, in either the dorsolateral or dorsomedial striatum (data not shown).

\section{Effect of methamphetamine on different types of spines}

The effect of prior treatment with methamphetamine was selective, in that it influenced the number of some types of spines, but not others. Figure 4A and B illustrates the four different types of spines that were quantified. For the dorsolateral striatum a two-way ANOVA resulted in a significant main effect of drug $(F=11.447, P<0.01)$, spine type $(F=207.061, \quad P<0.0001)$, and a drug-spine type interaction $(F=11.447, P<0.05)$. Figure $4 \mathrm{C}$ shows that prior exposure to methamphetamine significantly increased the number of 'thin' $(P<0.05)$ and 'mushroom' spines $(P<0.05)$, but had no effect on the number of 'stubby' $(P=0.453)$ or 'chubby' spines $(P=0.806)$. For the dorsomedial striatum a two-way ANOVA also resulted in a 

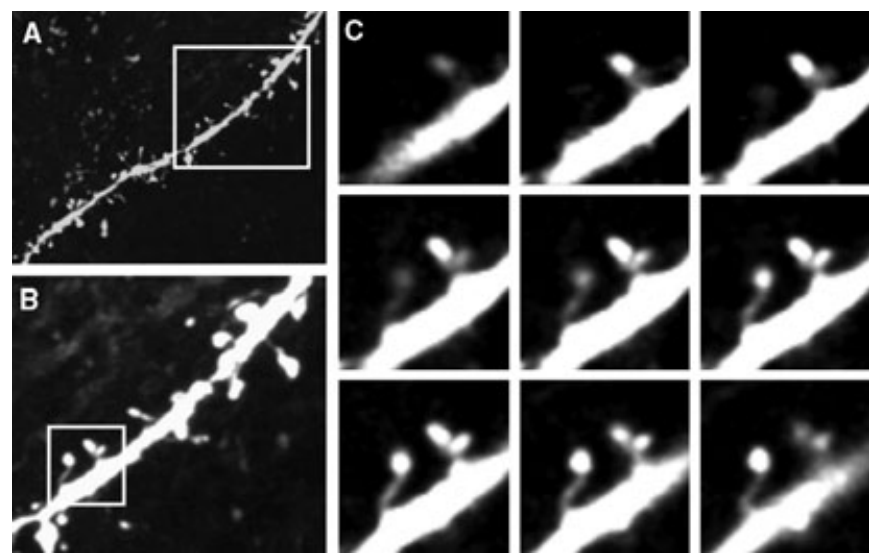

FIG. 2. Examples of images acquired via confocal microscopy following expression of GFP by Sindbis virus. (A) Confocal stack of a dendrite on a MSN in the caudate putamen. (B) Magnified view of the dendrite from the boxed area in (A). Notice the clarity and ability to distinguish the majority of spines. (C) Higher magnification of images in a confocal stack from the boxed area in (B). These types of images were used for guidance when the ability to separate spines in close proximity became ambiguous.
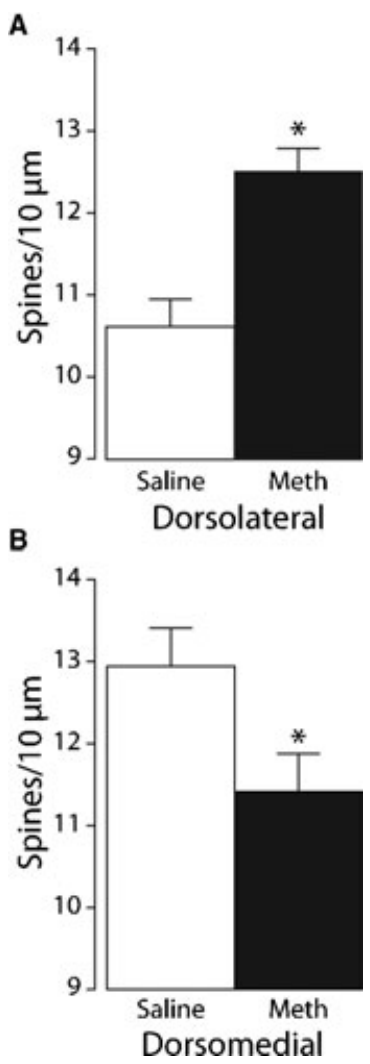

FIG. 3. Effects of repeated methamphetamine administration on the density of dendritic spines on MSNs in the dorsomedial (B) and dorsolateral (A) caudate putamen. Repeated injections of methamphetamine decreased spine density in the dorsomedial striatum and increased spine density in the dorsolateral striatum. The asterisk denotes a significant difference as compared with salinetreated animals.

significant drug-spine type interaction $(F=2.782, P<0.05)$. In this region, prior methamphetamine only decreased the number of 'mushroom' spines $(P<0.05$; Fig. 4D), and there were no significant differences between the methamphetamine and saline groups in the number of 'stubby' $(P=0.172)$, 'thin' $(P=0.922)$ or 'chubby' spines $(P=0.374)$.

\section{Discussion}

Sindbis virus was employed to express GFP in neurons to study the effects of repeated treatment with methamphetamine on dendritic structure in the dorsal striatum, 3 months after the last drug injection. Although this technology has been used previously to visualize dendrites (Maletic-Savatic et al., 1999; Lendvai et al., 2000), to our knowledge this is the first application of the technique to study experience-dependent structural plasticity in the intact, adult animal (cf. Lendvai et al., 2000). This technique provided excellent visualization of dendrites and different types of spines and, thus, it is an alternative to Golgi-staining for this purpose. Repeated treatment with methamphetamine increased the density of mushroom and thin spines on MSNs in the dorsolateral striatum, confirming and extending a previous report in which Golgi-Cox staining was used in animals treated with D-amphetamine (Li et al., 2003). Therefore, the persistent effect of repeated treatment with amphetamine on spine density is not specific to Golgi-Cox staining in either the dorsal striatum (present study) or the nucleus accumbens (Lee et al., 2006), and D-amphetamine and methamphetamine produce similar effects on spine density in the dorsolateral striatum.

In contrast to an increase in spine density in the dorsolateral striatum, there was a significant decrease in the density of mushroom spines in the immediately adjacent dorsomedial striatum. Of course, electron microscopic (EM) studies are required to determine if either the increase or decrease in spines seen here are associated with changes in morphologically identifiable synapses, and electrophysiological studies to determine the functional characteristics of such synapses. It is encouraging, however, that one recent EM study has shown that the repeated intermittent administration of cocaine is accompanied by a persistent increase in asymmetric synapses in the core of the nucleus accumbens (Berlanga et al., 2006). This is consistent with research on other forms of experience-dependent plasticity, which suggests that the kinds of changes in spines described here are accompanied by both changes in synaptogenesis and in cell signaling (Greenough et al., 1990; Kolb \& Whishaw, 1998; Woolley, 1999; Trachtenberg et al., 2002). For example, increases in synaptic strength, such as those modeled by long-term potentiation (LTP), are accompanied by the growth of new spines and increases in spine density (Engert \& Bonhoeffer, 1999; Ivanco et al., 2000), whereas the inhibition of LTP prevents new spine formation (Engert \& Bonhoeffer, 1999). Long-term depression (LTD)-inducing protocols, which reduce synaptic strength, result in spine loss (Nagerl et al., 2004). Importantly, the formation and retraction of spines has also been shown to be accompanied by synapse formation and elimination (Trachtenberg et al., 2002).

In the dorsal striatum the distal dendrites of MSNs are thought to be the major site of convergence of glutamatergic inputs from the neocortex, thalamus, amygdala and hippocampus, and ascending dopaminergic inputs from the substantia nigra (Smith \& Bolam, 1990; Gerfen \& Wilson, 1996; Voorn et al., 2004). Approximately $90 \%$ of glutamatergic inputs onto MSNs are thought to make asymmetric synapses on the head of spines. It is important therefore that we found significant changes in the number of mushroom spines, because these are thought to reflect mature spines containing functional AMPA receptors (Takumi et al., 1999; Matsuzaki et al., 2001; Kasai et al., 2003). Indeed, there is a positive correlation between spine head volume and the expression of functional AMPA receptors (Matsuzaki 


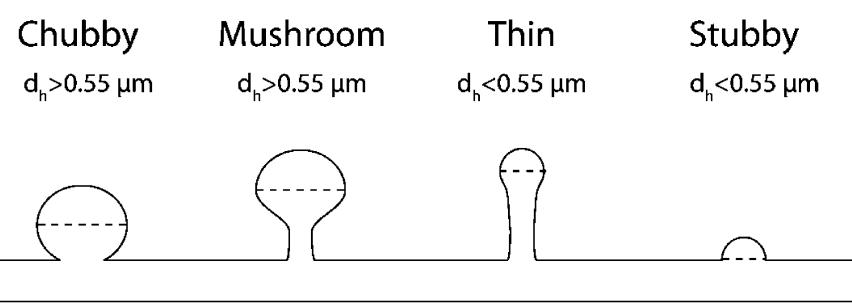

B

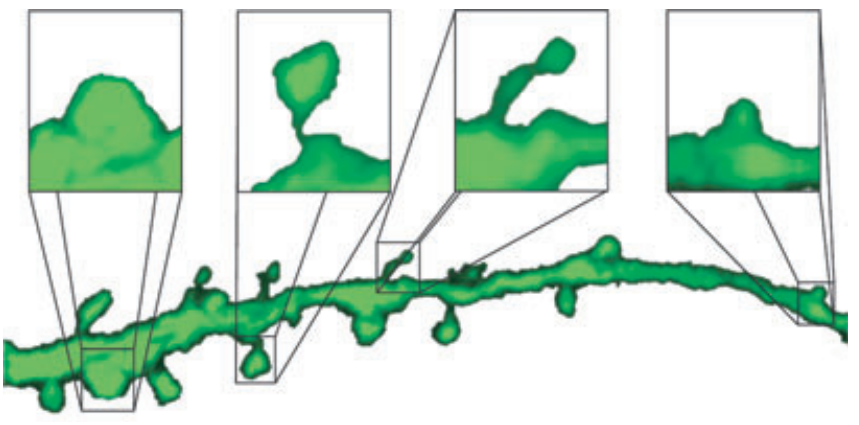

C
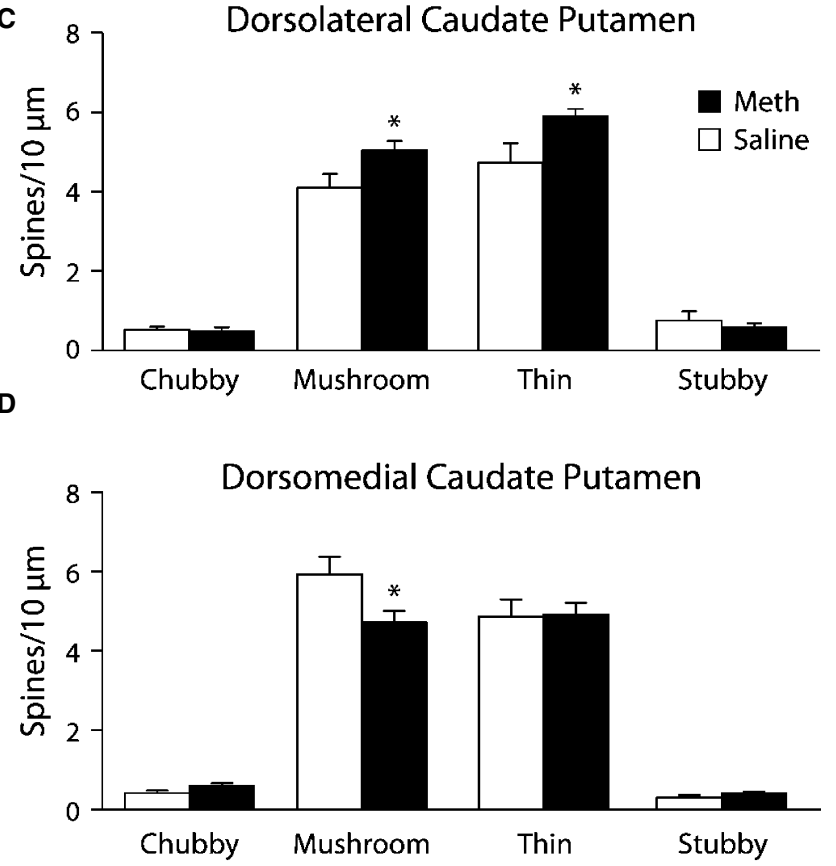

FIG. 4. Effects of repeated methamphetamine administration on the types of dendritic spines on MSNs in the dorsomedial and dorsolateral caudate putamen. (A) Criteria applied to separate spines in four morphologically distinct groups. (B) Example of a dendrite that contains all four spine types. (C) Repeated injections of methamphetamine significantly increased the number of mushroom and thin type spines in the dorsolateral caudate putamen and (D) decreased the number of mushroom type spines in the dorsomedial caudate putamen. The asterisk denotes a significant difference as compared with saline-treated animals.

et al., 2004). Thus, the available data support the speculation that the changes in mushroom spines may reflect an increase in the influence of corticostriatal (and perhaps other afferent) control over the dorsolateral striatum, and a decrease in corticostriatal influence over the dorsomedial striatum. Furthermore, the additional thin spines seen in the dorsolateral striatum may represent potential sites for future plasticity, as such spines are thought to contain $N$-methyl-D-aspartate (NMDA) but not AMPA receptors (Kasai et al., 2003). Perhaps the increase in thin spines provides a foundation for subsequent plasticity in the dorsolateral striatum, which could include the facilitation of subsequent habit learning produced by past exposure to amphetamine (Nelson \& Killcross, 2006).

When animals initially learn that an action reliably produces a desired outcome they tend to repeat that action, and initially their behavior is controlled by explicit anticipation of the outcome or goal (Dickinson \& Balleine, 1994). The dorsomedial (and ventral) striatum is especially important for such goal-directed behavior (Balleine, 2005; Yin \& Knowlton, 2006). However, under some conditions some behaviors become more and more 'automatic' and habitual with repetition, and are guided by implicit S-R associations dissociated from the actual outcome. It has been known for some time that the dorsal striatum is especially important for habit (S-R) learning (Mishkin et al., 1984), and more recent research suggests this is specifically a function of the dorsolateral striatum (Yin et al., 2004, 2006). This transition in the control of behavior from actionoutcome associations to $\mathrm{S}-\mathrm{R}$ associations, and presumably from the dorsomedial (and ventral) to the dorsolateral striatum has been hypothesized to occur in association with the development of habits in addicts (Tiffany, 1990; Berke \& Hyman, 2000; Everitt et al., 2001). Although we continue to maintain that S-R habits do not explain the 'core' features of addiction (Robinson \& Berridge, 2003), there is no doubt that 'automatized' habits, which are especially evident during the act of consuming drugs, represent an interesting symptom of addiction, or that drugs promote the development of habits. For example, behavior rewarded by the receipt of drugs becomes habitual more readily than behavior rewarded by food, as indicated by insensitivity to reward devaluation (Dickinson et al., 2002; Miles et al., 2003). Furthermore, the dorsolateral striatum is important for the ability of drug-associated cues to maintain drugseeking behavior under second-order schedules of reinforcement (Ito et al., 2002; Vanderschuren et al., 2005), and the inactivation of the dorsolateral striatum attenuates the resumption (reinstatement) of cocaine-seeking after a period of abstinence (Fuchs et al., 2006). In addition, in humans cue-induced drug craving is associated with increased dopamine neurotransmission in the putamen (dorsolateral striatum) (Volkow et al., 2006). It is especially important that even past treatment with experimenter-administered amphetamine (as used here) results in 'a rapid progression from goal-directed to habit-based responding' (Nelson \& Killcross, 2006, p. 3805). This phenomenon may be related to the development of progressively more stereotyped patterns of behavior that are produced by repeated treatment with psychostimulant drugs (Robinson \& Becker, 1986), which has also been associated with adaptations in the dorsal striatum (Paulson \& Robinson, 1995; Canales \& Graybiel, 2000; Li et al., 2003), as well as other brain regions. Finally, the long-term self-administration of cocaine has been shown to progressively engage the dorsolateral striatum (Porrino et al., 2004).

We hypothesize therefore that the psychostimulant drug-induced increase in spines in the dorsolateral striatum (reflecting increased excitatory input) may represent a structural manifestation of increased control over behavior by the dorsolateral striatum, which could account for why exposure to psychostimulant drugs facilitates the later development of S-R habits (Miles et al., 2003; Nelson \& Killcross, 2006). If there is also a reduction in spines in the more posterior dorsomedial striatum, the specific striatal region that has been implicated in action-outcome learning (Yin et al., 2005a,b), akin to 
the reduction in the more anterior dorsomedial striatum described here, this could reflect the structural manifestation of reduced control over behavior by this brain region, leading to a decrease in the control over behavior by outcomes. Alternatively, the changes in the dorsomedial striatum described here could also reflect decreased control of the dorsolateral striatum by the dorsomedial striatum, which is thought to occur via a 'spiraling' network of connections between the tegmentum and striatum that proceeds from ventromedial to more dorsolateral sectors of the system (Haber et al., 2000).

It is intriguing that methamphetamine had such different effects on spine density in the dorsolateral vs. dorsomedial striatum, but this is consistent with reports that these two regions show different forms of synaptic plasticity as a function of activation of corticostriatal inputs. For example, high-frequency stimulation of cortical projection neurons in slice preparations has been reported to induce LTP in the dorsomedial striatum, but LTD in the dorsolateral striatum (Partridge et al., 2000; Smith et al., 2001). However, the nature of the synaptic change is modulated by dopamine. A protocol of high-frequency stimulation that normally induces LTD in striatal slices produces LTP if the highfrequency stimulation is paired with the application of dopamine (Wickens et al., 1996; Reynolds \& Wickens, 2000). In the present study amphetamine was given in a relatively novel environment, which strongly activates the neocortex and influences gene expression in the dorsal striatum via corticostriatal fibers (Badiani et al., 1998; Ferguson \& Robinson, 2004), and would also release large amounts of dopamine. The present results suggest that this combination of events induces different forms of synaptic plasticity in the dorsolateral vs. dorsomedial striatum, but the mechanisms by which this occurs are unknown.

Indeed, very little is known about the mechanisms of synapse formation in adulthood, and even less on how exposure to drugs of abuse influences spine formation. One possibility is suggested by reports that cocaine restructures actin binding protein complexes, increasing actin recycling (Toda et al., 2006). However, this effect is seen in the nucleus accumbens but not dorsal striatum, and is associated primarily with filopodia formation. But repeated amphetamine and cocaine increase the number of presumably mature mushroom spines in both the dorsolateral striatum (present study) and the nucleus accumbens (Lee et al., 2006). Another possibility comes from studies showing that repeated exposure to cocaine persistently increases delta FosB expression. This protein not only modulates the effects of psychostimulant drugs (Nestler, 2001), but one downstream target of delta FosB is Cdk5, and a number of molecules involved in the regulation of spine morphology are substrates for Cdk5 (Bibb, 2003; Cheung et al., 2006). Furthermore, inhibition of Cdk5 prevents cocaine-induced spine formation (Norrholm et al., 2003), and the type of MSNs that persistently express delta FosB after repeated cocaine are those that show a long-lasting increase in spine density (Lee et al., 2006). These latter studies have focused on the nucleus accumbens, but if this signaling cascade is also involved in structural plasticity in the dorsal striatum we would predict it is differentially activated in the dorsolateral vs. dorsomedial striatum. In studying mechanisms of drug experience-dependent plasticity, pooling tissue from these two subregions of the dorsal striatum is clearly not advisable. Indeed, a direct comparison of these two subregions may provide an excellent approach to unravel the complex signaling cascades involved in drug-induced spine formation and spine elimination.

\section{Acknowledgements}

This research was supported by a grant from the National Institute on Drug Abuse (R01 DA13398 and F31 DA01920) to T.E.R. and J.J. We thank Don Backos and Peng Wei Yang for technical assistance.

\section{Abbreviations}

GFP, green fluorescent protein; LTD, long-term depression; LTP, long-term potentiation; MSNs, medium spiny neurons; PBS, phosphate-buffered saline; $\mathrm{S}-\mathrm{R}$, stimulus-response.

\section{References}

Badiani, A., Oates, M.M., Day, H.E.W., Watson, S.J., Akil, H. \& Robinson, T.E. (1998) Amphetamine-induced behavior, dopamine release, and c-fos mRNA expression: modulation by environmental novelty. J. Neurosci., 18, 10579-10593.

Balleine, B.W. (2005) Neural bases of food-seeking: affect, arousal and reward in corticostriatolimbic circuits. Physiol. Behav., 86, 717-730.

Berke, J.D. \& Hyman, S.E. (2000) Addiction, dopamine, and the molecular mechanisms of memory. Neuron, 25, 515-532.

Berlanga, M.L., Lim, H.Y., Floyd, C.E., Garces, J., Mendenhall, J.M., Lyons, C.L. \& Alcantara, A.A. (2006) Synaptic rewiring in the nucleus accumbens core is associated with cocaine-induced behavioral sensitization. Soc. Neurosci. Abstr., 482.10.

Bibb, J.A. (2003) Role of Cdk5 in neuronal signaling, plasticity, and drug abuse. Neurosignals, 12, 191-199.

Canales, J.J. \& Graybiel, A.M. (2000) A measure of striatal function predicts motor stereotypy. Nat. Neurosci., 3, 377-383.

Cheung, Z.H., Fu, A.K. \& Ip, N.Y. (2006) Synaptic roles of Cdk5: implications in higher cognitive functions and neurodegenerative diseases. Neuron, 50, 13-18.

Dickinson, A. \& Balleine, B. (1994) Motivational control of goal-directed action. Anim. Learn. Behav., 22, 1-18.

Dickinson, A., Wood, N. \& Smith, J.W. (2002) Alcohol seeking by rats: action or habit? Q. J. Exp. Psychol. B, 55, 331-348.

Engert, F. \& Bonhoeffer, T. (1999) Dendritic spine changes associated with hippocampal long-term synaptic plasticity. Nature, 399, 66-70.

Everitt, B.J., Dickinson, A. \& Robbins, T.W. (2001) The neuropsychological basis of addictive behaviour. Brain Res. Rev., 36, 129-138.

Ferguson, S.M. \& Robinson, T.E. (2004) Amphetamine-evoked gene expression in striatopallidal neurons: regulation by corticostriatal afferents and the ERK/MAPK signaling cascade. J. Neurochem., 91, 337-348.

Fuchs, R.A., Branham, R.K. \& See, R.E. (2006) Different neural substrates mediate cocaine seeking after abstinence versus extinction training: a critical role for the dorsolateral caudate-putamen. J. Neurosci., 26, 35843588 .

Gerfen, C.R. \& Wilson, C.J. (1996) The basal ganglia. In Swanson, L.W., Bjorklund, A. \& Hökfelt, T. (Eds), Handbook of Chemical Neuroanatomy: Integrated Systems of the CNS, Part III, Vol. 12. Elsevier, Amsterdam, pp. $371-468$.

Greenough, W.T., Withers, G.S. \& Wallace, C.S. (1990) Morphological changes in the nervous system arising from behavioral experience: what is the evidence that they are involved in learning and memory? In Squire, L.R. \& Lindenlaub, E. (Eds), The Biology of Memory, Symposia Medica Hoechst. F.K. Schattauder, New York, pp. 159-185.

Haber, S.N., Fudge, J.L. \& McFarland, N.R. (2000) Striatonigrostriatal pathways in primates form an ascending spiral from the shell to the dorsolateral striatum. J. Neurosci., 20, 2369-2382.

Hamamura, T., Akiyama, K., Akimoto, K., Kashihara, K., Okumura, K., Ujike, H. \& Otsuki, S. (1991) Co-administration of either a selective D1 or D2 dopamine antagonist with methamphetamine prevents methamphetamine-induced behavioral sensitization and neurochemical change, studied by in vivo intracerebral dialysis. Brain Res., 546, 40-46.

Harris, K.M., Jensen, F.E. \& Tsao, B. (1992) Three-dimensional structure of dendritic spines and synapses in rat hippocampus (CA1) at postnatal day 15 and adult ages: implications for the maturation of synaptic physiology and long-term potentiation. J. Neurosci., 12, 2685-2705.

Hebb, D.O. (1949) The Organization of Behavior. John Wiley, New York.

Ito, R., Dalley, J.W., Robbins, T.W. \& Everitt, B.J. (2002) Dopamine release in the dorsal striatum during cocaine-seeking behavior under the control of a drug-associated cue. J. Neurosci., 22, 6247-6253.

Ivanco, T.L., Racine, R.J. \& Kolb, B. (2000) Morphology of layer III pyramidal neurons is altered following induction of LTP in sensorimotor cortex of the freely moving rat. Synapse, 37, 16-22.

Kasai, H., Matsuzaki, M., Noguchi, J., Yasumatsu, N. \& Nakahara, H. (2003) Structure-stability-function relationships of dendritic spines. Trends Neurosci., 26, 360-368.

Kolb, B., Gorny, G., Li, Y., Samaha, A.N. \& Robinson, T.E. (2003) Amphetamine or cocaine limits the ability of later experience to promote 
structural plasticity in the neocortex and nucleus accumbens. Proc. Natl Acad. Sci. USA, 100, 10523-10528.

Kolb, B. \& Whishaw, I.Q. (1998) Brain plasticity and behavior. Annu. Rev. Psychol., 49, 43-64.

Lee, K.W., Kim, Y., Kim, A.M., Helmin, K., Nairn, A.C. \& Greengard, P. (2006) Cocaine-induced dendritic spine formation in D1 and D2 dopamine receptor-containing medium spiny neurons in nucleus accumbens. Proc. Natl Acad. Sci. USA, 103, 3399-3404.

Lendvai, B., Stern, E.A., Chen, B. \& Svoboda, K. (2000) Experiencedependent plasticity of dendritic spines in the developing rat barrel cortex in vivo. Nature, 404, 876-881.

Li, Y., Kolb, B. \& Robinson, T.E. (2003) The location of persistent amphetamine-induced changes in the density of dendritic spines on medium spiny neurons in the nucleus accumbens and caudate-putamen. Neuropsychopharmacology, 28, 1082-1085.

Maletic-Savatic, M., Malinow, R. \& Svoboda, K. (1999) Rapid dendritic morphogenesis in CA1 hippocampal dendrites induced by synaptic activity. Science, 283, 1923-1927.

Matsuzaki, M., Ellis-Davies, G.C., Nemoto, T., Miyashita, Y., Iino, M. \& Kasai, H. (2001) Dendritic spine geometry is critical for AMPA receptor expression in hippocampal CA1 pyramidal neurons. Nat. Neurosci., 4, 10861092.

Matsuzaki, M., Honkura, N., Ellis-Davies, G.C. \& Kasai, H. (2004) Structural basis of long-term potentiation in single dendritic spines. Nature, 429, 761766.

Miles, F.J., Everitt, B.J. \& Dickinson, A. (2003) Oral cocaine seeking by rats: action or habit? Behav. Neurosci., 117, 927-938.

Mishkin, M., Malamut, B. \& Bachevalier, J. (1984) Memories and habits: two neural systems. In Lynch, G., McGaugh, J.L. \& Weinberger, N.M. (Eds), Neurobiology of Learning and Memory. Guilford Press, New York, pp. 65-77.

Nagerl, U.V., Eberhorn, N., Cambridge, S.B. \& Bonhoeffer, T. (2004) Bidirectional activity-dependent morphological plasticity in hippocampal neurons. Neuron, 44, 759-767.

Nelson, A. \& Killcross, S. (2006) Amphetamine exposure enhances habit formation. J. Neurosci., 26, 3805-3812.

Nestler, E.J. (2001) Molecular basis of long-term plasticity underlying addiction. Nat. Rev. Neurosci., 2, 119-128.

Norrholm, S.D., Bibb, J.A., Nestler, E.J., Ouimet, C.C., Taylor, J.R. \& Greengard, P. (2003) Cocaine-induced proliferation of dendritic spines in nucleus accumbens is dependent on the activity of cyclin-dependent kinase-5. Neuroscience, 116, 19-22.

Partridge, J.G., Tang, K.C. \& Lovinger, D.M. (2000) Regional and postnatal heterogeneity of activity-dependent long-term changes in synaptic efficacy in the dorsal striatum. J. Neurophysiol., 84, 1422-1429.

Paulson, P.E., Camp, D.M. \& Robinson, T.E. (1991) The time course of transient behavioral depression and persistent behavioral sensitization in relation to regional brain monoamine concentrations during amphetamine withdrawal in rats. Psychopharmacology, 103, 480-492.

Paulson, P.E. \& Robinson, T.E. (1995) Amphetamine-induced time-dependent sensitization of dopamine neurotransmission in the dorsal and ventral striatum: a microdialysis study in behaving rats. Synapse, 19, 56-65.

Porrino, L.J., Lyons, D., Smith, H.R., Daunais, J.B. \& Nader, M.A. (2004) Cocaine self-administration produces a progressive involvement of limbic, association, and sensorimotor striatal domains. J. Neurosci., 24, 3554-3562.

Ramón y Cajal, S. (1894) La fine structure des centres nerveux. Proc. R. Soc. Lond., 55, 444-468.

Reynolds, J.N. \& Wickens, J.R. (2000) Substantia nigra dopamine regulates synaptic plasticity and membrane potential fluctuations in the rat neostriatum, in vivo. Neuroscience, 99, 199-203.
Robinson, T.E. (1984) Behavioral sensitization: characterization of enduring changes in rotational behavior produced by intermittent injections of amphetamine in male and female rats. Psychopharmacology (Berl.), 84, 466-475.

Robinson, T.E. \& Becker, J.B. (1986) Enduring changes in brain and behavior produced by chronic amphetamine administration: a review and evaluation of animal models of amphetamine psychosis. Brain Res. Rev., 11, 157-198.

Robinson, T.E. \& Berridge, K.C. (2003) Addiction. Annu. Rev. Psychol., 54, 25-53.

Robinson, T.E. \& Kolb, B. (2004) Structural plasticity associated with exposure to drugs of abuse. Neuropharmacology, 47 (Suppl. 1), 33-46.

Rosenzweig, M.R. (2003) Effects of differential experience on the brain and behavior. Dev. Neuropsychol., 24, 523-540.

Smith, A.D. \& Bolam, J.P. (1990) The neural network of the basal ganglia as revealed by the study of synaptic connections of identified neurones. Trends Neurosci., 13, 259-265.

Smith, R., Musleh, W., Akopian, G., Buckwalter, G. \& Walsh, J.P. (2001) Regional differences in the expression of corticostriatal synaptic plasticity. Neuroscience, 106, 95-101.

Takumi, Y., Ramirez-Leon, V., Laake, P., Rinvik, E. \& Ottersen, O.P. (1999) Different modes of expression of AMPA and NMDA receptors in hippocampal synapses. Nat. Neurosci., 2, 618-624.

Tiffany, S.T. (1990) A cognitive model of drug urges and drug-use behavior: role of automatic and nonautomatic processes. Psychol. Rev., 97, 147-168.

Toda, S., Shen, H.W., Peters, J., Cagle, S. \& Kalivas, P.W. (2006) Cocaine increases actin cycling: effects in the reinstatement model of drug seeking. J. Neurosci., 26, 1579-1587.

Trachtenberg, J.T., Chen, B.E., Knott, G.W., Feng, G., Sanes, J.R., Welker, E. \& Svoboda, K. (2002) Long-term in vivo imaging of experience-dependent synaptic plasticity in adult cortex. Nature, 420, 788-794.

Vanderschuren, L.J., Di Ciano, P. \& Everitt, B.J. (2005) Involvement of the dorsal striatum in cue-controlled cocaine seeking. J. Neurosci., 25, 86658670 .

Volkow, N.D., Wang, G.J., Telang, F., Fowler, J.S., Logan, J., Childress, A.R., Jayne, M., Ma, Y. \& Wong, C. (2006) Cocaine cues and dopamine in dorsal striatum: mechanism of craving in cocaine addiction. J. Neurosci., 26, 65836588.

Voorn, P., Vanderschuren, L.J., Groenewegen, H.J., Robbins, T.W. \& Pennartz, C.M. (2004) Putting a spin on the dorsal-ventral divide of the striatum. Trends Neurosci., 27, 468-474.

Wickens, J.R., Begg, A.J. \& Arbuthnott, G.W. (1996) Dopamine reverses the depression of rat corticostriatal synapses which normally follows highfrequency stimulation of cortex in vitro. Neuroscience, $\mathbf{7 0}, 1-5$.

Woolley, C.S. (1999) Electrophysiological and cellular effects of estrogen on neuronal function. Crit. Rev. Neurobiol., 13, 1-20.

Yin, H.H. \& Knowlton, B.J. (2006) The role of the basal ganglia in habit formation. Nat. Rev. Neurosci., 7, 464-476.

Yin, H.H., Knowlton, B.J. \& Balleine, B.W. (2004) Lesions of dorsolateral striatum preserve outcome expectancy but disrupt habit formation in instrumental learning. Eur. J. Neurosci., 19, 181-189.

Yin, H.H., Knowlton, B.J. \& Balleine, B.W. (2005a) Blockade of NMDA receptors in the dorsomedial striatum prevents action-outcome learning in instrumental conditioning. Eur. J. Neurosci., 22, 505-512.

Yin, H.H., Knowlton, B.J. \& Balleine, B.W. (2006) Inactivation of dorsolateral striatum enhances sensitivity to changes in the action-outcome contingency in instrumental conditioning. Behav. Brain Res., 166, 189-196.

Yin, H.H., Ostlund, S.B., Knowlton, B.J. \& Balleine, B.W. (2005b) The role of the dorsomedial striatum in instrumental conditioning. Eur. J. Neurosci., 22, 513-523. 